\title{
Genetic Parameter Estimates for Growth and Reproductive Trait of Sheep for Genetic Improvement and Designing Breeding Program in Ethiopia: A Review
}

\author{
Shigdaf Mekuriaw ${ }^{1^{*}}$, Aynalem Haile ${ }^{2}$ \\ ${ }^{1}$ Andassa Livestock Research Center, Bahir Dar, Ethiopia \\ ${ }^{2}$ International Center for Agricultural Research in the Dry Areas, Addiss Abeba, Ethiopia \\ Email: ${ }^{*}$ shigdafmekuriaw@yahoo.com
}

Received 3 June 2014; revised 6 July 2014; accepted 13 August 2014

Copyright (C) 2014 by authors and OALib.

This work is licensed under the Creative Commons Attribution International License (CC BY). http://creativecommons.org/licenses/by/4.0/

(c) (i) Open Access

\section{Abstract}

Small ruminants in general and sheep in particular are important investments in the highlands of Ethiopia. Genetic and environmental improvements offer an opportunity to increase production from existing animal resources. Development of effective genetic evaluation and improvement programs requires knowledge of the genetic parameters (genetic variance of each trait and covariance among traits) for economically important production traits. Direct heritability estimates for birth weight of Afar sheep (0.13 to 0.38) and BHS (0.20 to 0.58$)$ using univariate analysis; for Horro sheep $(0.18-0.32)$ and Menz sheep $(0.46)$ by fitting a multi-trait individual animal model. The weaning weight direct heritability estimates for sheep in Ethiopia, Afar sheep (0.11 - 0.37) and BHS (0.00 - 0.29), Horro sheep (0.10 - 0.26) and Menz sheep (0.48). Fertility, litter size and lamb survival are the components of the overall ewe reproduction traits. It appears that heritability estimates of these traits are rather low and reflect the generally small genetic variance for most reproductive traits. Moreover, litter size has a higher heritability $(0.16-0.19)$ than the other components traits like fertility $(0.10)$ and survival rate $(0.07-0.09)$ for Awassi sheep breed. Genetic correlations among most pairs of traits were higher than the corresponding phenotypic correlations. Live weights measured at successive ages were highly correlated, ranging from 0.61 to 0.98 for Menz sheep. Genetic correlations were also high between pre-weaning daily gain and weaning weight for Afar and BHS sheep. Except for Blackhead Somali sheep breed which has high genetic correlation between birth weight and weaning weight, Horro, Menz and Afar sheep have high genetic correlation between post-weaning weight traits. This indicates that selection for one trait improves the other trait in those sheep breeds. In order to develop alternative improvement program for sheep breeds in Ethiopia, estimates of genetic parameters for economical growth and reproduction traits should be done by designing experiments since the information on most sheep

\footnotetext{
*Corresponding author.
} 


\title{
breed is nonexistence.
}

\section{Keywords}

\author{
Genetic Parameter, Sheep Breed, Heritability, Correlation and Breeding Program
}

\author{
Subject Areas: Agricultural Science, Genetics
}

\section{Introduction}

Small ruminants play a major role for strengthening the backbone of rural economy; especially sheep are integral to the cultural, social and economic livelihood of the rural farmers [1]. The profitability of a sheep production system is determined by both fertility and production traits [2]. Productivity in any sheep enterprise where meat is the main product can be measured in terms of total weight of lambs weaned per ewe. This trait depends on fertility, litter size, weight (growth) of individual lambs, mothering ability and survival [3].

Small ruminants in general and sheep in particular are important investments in the highlands of Ethiopia. Efforts to improve the productivity of indigenous sheep have been part of the country's research undertakings [4]. Characterization of Ethiopian sheep breeds has been done by different studies. But there is no adequate information on estimate of genetic parameters for some economically important traits which will help to design appropriate selection programmes. However, most studies on indigenous sheep have so far concentrated on evaluating the breeds based only on phenotypic performance. For accurate genetic evaluation and selection, estimates of genetic parameters for traits of importance should be known. In this regard, growth and reproductive traits are important, as they significantly influence the profitability of any sheep production enterprises [4] and for prediction of the response to selection [5].

Genetic and environmental improvements offer an opportunity to increase production from existing animal resources. The genetic characterization of local breeds is of paramount importance, not only for conservation purposes but also for the definition of breeding objectives and breeding programmes [6]. Since productivity of the local sheep breeds are low due to the fact that animals are largely naturally selected for survival under suboptimal environments. Genetic improvement could also contribute to raise productivity from this system. Due to small flock sizes, genetic parameters which are required for genetic improvement have to be generated from onstation data where management is kept as close as possible to that existing under farm conditions [3]. When records are nonexistent and breeds are not well defined in the field, information could be obtained from research station information where pedigree and performance records have been kept [6].

Furthermore, development of breeding objectives and effective genetic improvement programs require knowledge of the genetic variation among economically important traits and accurate estimates of heritability, repeatability and genetic correlations of economically important traits [7] [8]. Due to this reason, this paper was initiated to review the available work encountering the genetic parameters estimated for growth and reproductive trait of sheep in Ethiopia.

\section{Methodologies for Genetic Parameter Estimation}

Estimating genetic parameters for various livestock traits has been a main topic of animal breeding during the past half century [9]. Advances in statistical animal breeding and broadening its range of application and traits of interest provide great opportunities for animal agriculture. Genetic parameters were estimated based on the most appropriate model from both logit and probit analyses [3]. Variance and co-variance estimates from the analysis were used to calculate the different important genetic parameters such as heritability, repeatability and genetic correlations [10]. The choice of model is also a matter of deciding which gives the most precise estimate for the same total of animals measured with the given facility. Forexamplel, low heritability are more efficiently estimated from the half sib correlation than estimated by offspring-parent regressions eventhough, there is one difficulty with offspring-parent regression method because data from two consecutive generations is needed [11] Some of the important models used for genetic parameter estimation are discussed below.

Animal model is extensively used for predicting genetic values and estimating genetic parameters, because 
the optimum combined use of all relationships and performances improves accuracy. However, despite the theoretical advantages of this model, some data and model conditions can affect the validity and precision of the estimation of variance components [12]. According to [13], Animal models resulted in slightly greater estimates of heritability for fertility than did sire models but ovulation rate heritability estimates from sire models were much greater than estimates form animal models. The genetic and non-genetic effects for each of the weights and growth traits were analyzed by using the animal model. According to [14], direct and maternal heritabilities and covariance between direct and maternal effects were initially estimated using single trait analysis. In most of the tropics, conventional finite animal models have been used in the analysis of growth and reproduction traits. These models include univariate and multivariate models that are useful to estimate genetic parameters of one trait at a time and several traits at once. In multivariate animal models, it is hard to estimate genetic parameters having a sampling correlation. However, if we ignore this, it may result in wrong estimates [9].

Many methodologies for categorical traits have been investigated in animal breeding, which can generally be classified into two groups, linear and non-linear models. In the linear models, least squares, generalized least squares and the log-linear model are procedures that have been used in the estimation of fixed effects for categorical data [15]. Nonlinear models were the threshold, Poisson, and negative binomial. Threshold sire and animal models were used with all traits. Litter size and ovulation rate were analyzed also with Poisson sire and animal models and with negative binomial sire and animal models. Variance components for linear models were estimated using Restricted Maximum Likelihood while in the threshold, Poisson and negative binomial, they were estimated using approximate marginal maximum likelihood. Poisson and negative binomial analyses yielded results difficult to interpret due to problems in variance component estimation. Genetic parameters were also estimated by Restricted Maximum Likelihood (REML) method using Derivative Free Restricted Maximum Likelihood (DFREML) method [14]. Maximum likelihood is used to estimate variance components. Even though maximum likelihood estimators have good properties, they have limitations in that the distribution of the data is assumed to be known, and that estimators are biased when fixed effects are managed as if they were known but these limitations could be removed by Restricted Maximum Likelihood [9].

In the case of REML, it is computationally difficult due to the inverse of coefficient matrix. Therefore, [16] considered a derivative-free (DF) REML algorithm for the univariate analysis under an animal model. Even with REML and DFREML, there are still problems with data analysis due to the inverse of coefficient matrix. Therefore, more effective methods in view of computing time were developed. Method $\mathrm{R}$ estimates variance components by using the linear regression coefficient, that is, "R", of more accurate recent data on less accurate previous individual genetic predictions [17]. Method $\mathrm{R}$ is more effective than any other method in terms of the computing time and the data size. BLUP estimates of breeding values (EBV) were obtained by fitting multitrait individual animal model using ASREML equation:

$$
Y i=X i b i+Z i a i+e i,
$$

where $Y i$ is vector of observations for trait $i$, $b i$ denotes a vector of fixed effects for trait $i$, $a i$ is a vector of random animal effects for trait $i$, $e i$ is vector of random residual effects for trait $i$, and $X i$ and $Z i$ are incidence matrices relating records for trait $i$ to fixed and random animal effects, respectively. Heritability were calculated as $\sigma 2 a / \sigma 2 p$ using the estimated variance components.

On the other hand, Snelling et al. (1995) cited in [9] studied the comparison of several methods like animal model marginal maximum likelihood, sire model marginal maximum likelihood and animal model Method R. However, the differences among these methods did not substantially influence the rank of individual predictions. As a result, Method R is an efficient method in processing time even though not affecting the rank of predictions. However, it can give very biased estimates of variances when relationship matrices are not complete and selection has been practiced. As a result models that account for time variance are more appropriate.

Generally, the different values of genetic parameter estimated and reported by many authors were, due to working on different breeds of sheep in different countries, the number of observation used in the analysis, the correction for the non-genetic factors and the model used in the analysis and the types of models used [18].

\section{Genetic Parameter Estimate for Growth and Reproductive Traits}

Effective breeding programmes depend on the accuracy of genetic parameter estimates, which include heritability, repeatability and correlation between traits [19]. Much more information on heritability, repeatability and genetic correlation estimates for growth and reproductive trait were not available for sheep in Ethiopian. How- 
ever, the limited available information is discussed below.

\subsection{Heritability $\left(h^{2}\right)$}

Heritability is defined as the proportion of phenotypic variance that is due to heredity. It is a measure of the degree to which a trait is genetically determined. Although not all of the genetic contribution is included, essentially all which is available to a breeder who is trying to select within a breed is included. Obviously heritability is important among the several factors determining how much genetic improvement can be made in any trait [20].

If individual will be selected based on their phenotypic values, success in improvement can be predicted only from the knowledge of correspondence between phenotypic and the breeding values. This degree of correspondence is measured by heritability. Since the genotype values are determined by the additive effects of the genes, heritability estimation is necessary to obtain the best estimate of an individual's breeding value [11].

\subsubsection{Heritability for Growth Traits}

Body weight and rate of gain are among the most economically important and easily measured traits of sheep. Knowledge of the particular trait and phase of the animal growth, which is base for selection, is the most important once [15]. The potential for genetic improvement is largely depend on the genetic parameters of growth weight trait upon which selection may be applied [21].

\subsubsection{Birth Weight}

Direct heritability estimates for birth weight of Afar sheep ( 0.13 to 0.38 ) and BHS (0.20 to 0.58 ) using univariate analysis [10]; for Horro sheep (0.18 - 0.32) [7] and Menz sheep (0.46) by fitting a multi-trait individual animal model [22] and [23] in Table 1. The estimates of heritability for birth weight and early growth are generally low to medium, which is usually attributed to the importance of variation in dam effects, especially in milk production and to competition between litter mates [15].

Table 1. Heritability estimate for growth traits.

\begin{tabular}{|c|c|c|c|c|}
\hline Trait & Sheep breed & $h_{a}^{2}$ & $h_{m}^{2}$ & Source \\
\hline Birth weight & Afar sheep & $0.13-0.38$ & $0.02-0.21$ & Yibrah (2008) \\
\hline Birth weight & BHS sheep & $0.2-0.58$ & $0.06-0.46$ & Yibrah (2008) \\
\hline Birth weight & Horro sheep & $0.18-0.32$ & $0.12-0.23$ & Abegaz et al. (2002a) \\
\hline Birth weight & Menz sheep & 0.46 & & Solomon et al. (2007b) \\
\hline Weaning weight & Afar sheep & $0.11-0.37$ & $0.12-0.21$ & Yibrah (2008) \\
\hline Weaning weight & BHS sheep & $0.00-0.29$ & $0.15-0.20$ & Yibrah (2008) \\
\hline Weaning weight & Menz sheep & 0.48 & & Solomon et al. (2007b) \\
\hline Weaning weight & Horro sheep & $0.10-0.26$ & 0.19 & Abegaz et al. (2002a) \\
\hline Six-month weight & Afar sheep & $0.14-0.32$ & $0.04-0.23$ & Yibrah (2008) \\
\hline Six-month weight & BHS sheep & $0.00-0.43$ & $0.12-0.23$ & Yibrah (2008) \\
\hline Six-month weight & Menz sheep & 0.51 & & Solomon et al. (2007b) \\
\hline Six-month weight & Horro sheep & $0.16-0.26$ & 0.24 & Abegaz et al. (2002a) \\
\hline Yearling weight & Afar sheep & $0.21-0.28$ & $0.02-0.25$ & Yibrah (2008) \\
\hline Yearling weight & BHS sheep & $0.12-0.25$ & $0.00-0.20$ & Yibrah (2008) \\
\hline Yearling weight & Horro sheep & $0.23-0.31$ & 0.09 & Abegaz et al. (2002a) \\
\hline Yearling weight & Menz sheep & 0.56 & & Solomon et al. (2007b) \\
\hline Pre-weaning gain & Afar sheep & $0.08-0.30$ & $0.20-0.28$ & Yibrah (2008) \\
\hline Pre-weaning gain & BHS sheep & $0.00-0.19$ & $0.02-0.18$ & Yibrah (2008) \\
\hline Post-weaning gain & Afar sheep & 0.09 & $0.00-0.09$ & Yibrah (2008) \\
\hline Post-weaning gain & BHS sheep & 0.00 & $0.00-0.02$ & Yibrah (2008) \\
\hline
\end{tabular}




\subsubsection{Weaning Weight}

The weaning weight direct heritability estimates for sheep in Ethiopia; Afar sheep $(0.11-0.37)$ and BHS (0.00 0.29) [10]; Horro sheep (0.10 - 0.26) [7] and Menz sheep (0.48) [22] in Table 1. [24] suggested that a weight collected in early life should be adequate for use in selecting for the direct component of weaning weight in sheep.

\subsubsection{Post-Weaning Weights and Gains}

Even though, Heritability estimates for weight at six-month weight and yearling weight are rarely reported in literature [25], the post-weaning weight and growth gain heritabilities of some Ethiopian sheep breeds are summarized in Table 1. Their range value is in line with [26] summarized 42 estimates of heritabilities for postweaning weight up to 9 months of age for dual-purpose breeds and 15 estimates for meat breeds (0.03 to 0.49 for the dual-purpose breeds and 0.06 to 0.41 for meat breeds).

Post-weaning growth was more heritable than pre-weaning growth, probably because nutrition was not a limiting factor after weaning. Given these large heritabilities, it appears that mass selection for rapid growth would be effective [15]. The interests in heritabilities of the different weights are simply in choosing the most adequate weight to use as a selection criterion to improve growth to weaning [27]. A common finding is that more progress in weaning weight can be made by selection on post-weaning weight than on weaning weight itself, due to the higher direct heritability of the post-weaning weight and its high genetic correlation with direct components of weaning weight.

\subsection{Heritability for Reproductive Traits}

Fertility, litter size and lamb survival are the components of the overall ewe reproduction traits. It appears that heritability estimates of these traits are rather low and reflect the generally small genetic variance for most reproductive traits. Moreover, Litter size has a higher heritability $(0.16-0.19)$ than the other components traits like fertility (0.10) and survival rate (0.07 - 0.09) for Awassi sheep breed [8].

\subsubsection{Fertility}

Direct heritability estimates for fertility was 0.02 ; when service sire was considered as fixed effect, heritability estimate of fertility from a direct model increased to 0.06 [3].

The heritability estimate for fertility is very low throughout and it becomes much lower as a result of inclusion of service sire as a non-genetic random effect. The data were generated from a single sire controlled mating system where assignment of rams to a group was done randomly after initial choice of rams to be used as sires. Due to this there was a need to include the serving ram as a random effect to separate its contribution to variation in ewe fertility [3].

\subsubsection{Litter Size}

The importance of litter size is that an increase in the number of lambs weaned per ewe per year offers the greatest single opportunity for increasing the efficiency of any kind of sheep production. More studies have addressed to genetic improvement of this trait than any other sheep reproductive trait. A lot of genetic variation for litter size exists between and within breeds [15]. Heritability estimates of litter size for Horro sheep ranges from 0.06 under the repeatability of model to 0.17 under the sire model; the direct heritability was 0.11 [4]. Heritability of twinning for Horro sheep was estimated to be 0.15 and 0.07 for the direct additive and repeatability models [7] which is slightly higher than 0.06 to 0.11 estimated by [3].

Comparison of heritability estimates for productive and reproductive traits depict lower estimates for female reproductive than productive traits because female reproductive traits were highly influenced by the environment and it could be more improved through manipulation of production environment than selection [19]. The high heritability estimates for productive traits were due to the high genetic variances attributed to this traits implying possibility of improvement through selection. There was an increase in maternal heritability up to weaning after which the estimates decreased. Maternal heritability is a function of maternal variance that arises from the environment (dam effect). However at weaning when the lamb is separated from the dam, the maternal environment is withdrawn and thus the effects of this environment on variance declines as the lamb grows and becomes independent [28]. 


\subsubsection{Repeatability}

Repeatability measures the correlation between the repeated measurements of the same individual. It indicates the gain in accuracy that may be expected from the use of the mean multiple measurements instead of single measurement [11]. Basically repeatability value is greater than heritability value since repeatability estimates include the permanent maternal environmental variance in addition the additive genetic variance component [4].

\subsubsection{Repeatability for Growth Traits}

Repeatability estimates were 0.10 and 0.07 for birth weight and weaning weight under random and fixed sire models respectively for Horro sheep [3]. This result is lower for the repeatability estimates for growth traits range from $0.13-0.37$ for birth weight and 0.08 - 0.29 for weaning weight for Awassi sheep, however, the repeatability for adult body weight of ewes is higher (0.43) [8].

\subsubsection{Repeatability for Reproductive Traits}

Solomon [28] estimate the repeatability of twinning (0.16) for Horro sheep is higher than an estimated by [7] while repeatability of fertility was 0.02 and 0.08 when service sire was considered random and fixed. This repeatability of fertility value is lower than repeatability estimates of 0.10 to 0.17 from Finnsheep and Rambouillet sheep by linear sire animal models and threshold sire models [13]. The accuracy of repeatability estimates for reproductive traits were low than heritability estimates for selection improvement because repeatability is measures of correlation between performances of traits in different parity [29].

\subsection{Genetic Correlation among Traits}

The main causes of genetic correlation as stated by [30] are chiefly pleiotropic which is simply the property of genes affecting two or more traits. Linkage can also affect different traits and cause genetic correlation. In general, estimates of genetic correlation between any pair of traits suggest that selection for one trait can lead to an indirect genetic response in the other trait [11].

\subsubsection{Genetic Correlation among Growth Traits}

The correlations between direct and maternal additive genetic effects for birth weight, weaning weight, six-month weight and yearling weight were $0.64,0.42,0.002$ and 0.46 , respectively for Horro sheep [4]. The correlations between body weights at different ages decreased, as the time between measurements increased. Genetic correlations among most pairs of traits were higher than the corresponding phenotypic correlations. Live weights measured at successive ages were highly correlated, ranging from 0.61 to 0.98 for Menz sheep [22]. As shown in Table 2, genetic correlations were also high between pre-weaning daily gain and weaning weight for Afar and BHS sheep [10].

\subsubsection{Genetic Correlation among Reproductive Traits}

The genetic correlation of twinning with birth weight and weaning weight is 0.77 and 0.26 whereas for litter size at weaning with birth weight and weaning weight is 0.45 and 0.84 respectively for Horro sheep [3].

\subsection{Practical Utility of Genetic Parameters}

Generally production traits have been reported to have a medium to high repeatability and low to medium heritability hence there exists opportunity for improvement through selection. Genetic trends in performance traits are important in that they allow for the evaluation of the efficacy of selection and management schemes

\begin{tabular}{|c|c|c|c|c|c|c|c|}
\hline Breed & BW-WW & BW-6MW & BW-YW & WW-6MW & WW-YW & 6MW-YW & Source \\
\hline Afar sheep & 0.73 & 0.74 & 0.70 & 0.78 & 0.71 & 0.95 & Yibrah (2008) \\
\hline BHS sheep & 0.86 & 0.87 & 0.65 & 0.99 & 0.89 & 0.65 & Yibrah (2008) \\
\hline Horro sheep & 0.45 & 0.33 & 0.30 & 0.97 & 0.837 & 0.87 & Abegaz et al. (2002) \\
\hline Menz sheep & 0.51 & 0.52 & 0.49 & 0.82 & 0.69 & 0.81 & Solomon et al. (2007) \\
\hline
\end{tabular}

BW—Birth weight; WW—Weaning weight; 6MW—Six-month weight; YW—Yearling weight. 
[14]. The basic pre-requisite of any breed improvement program is a priori knowledge about the extent of variability, or more precisely, the additive genetic variability present within the population under study, association between traits and repeatability of a trait [20]. In practical utility of genetic parameters successful used on sheep genetic improvement in Ethiopia: growth and reproductive trait for Menz and Horo sheep [7] and [10].

\subsubsection{Genetic Improvement through Selection}

Estimates of genetic parameters suggest that there exists substantial additive genetic variability in the population. Moderate heritability estimates for studied traits, indicated that, improving programs for these trait must be included improving environmental conditions with genetic improvement to achieved results preferable [18]. The potential for genetic improvement is largely dependent on the heritability of the trait and its relationship with other traits of economic importance [15]. Estimates of genetic parameters and observed genetic trends confirm that selective breeding can lead to significant genetic improvement in Menz sheep [22].

\subsubsection{Growth Rate Improvement}

Most scientists agreed that selection for growth should be based on traits which can be measured early in the animal life. It is generally agreed that more progress in weaning weight can be made by selection on postweaning weight or gain than pre-weaning weight or gain, due to the higher direct heritability of the post-weaning weight and its high genetic correlation with the weaning trait in sheep [31]. The high genetic correlation between weight at six-month and weight at one year indicates that breeding rams could be selected at an earlier age of six months and selective breeding can lead to significant genetic improvement in Menz sheep [22].

According to [4], the medium to high direct and maternal heritability estimates obtained for birth weight and yearling weight indicate that in Horro sheep faster genetic improvement through selection is possible for these traits and it should consider both (direct and maternal) heritability estimates. However, since the direct-maternal genetic covariances were found to be negative, caution should be made in making selection decisions.

The higher genetic correlations indicate selection for indirect selection for a correlated trait, practical implication of this is that indirect selection for late traits (yearling weight) through selection for earlier traits (e.g. weaning or six-month weight) [4]. In Afar and BHS sheep breeds the genetic correlations between birth weight and weaning, six-month and yearling weights are high. This implies that genetic improvement of birth weight of lambs can improve their growth performance at later age. The birth weight also is important in improving survival of lambs. Therefore selection of Afar and blackhead lambs for birth weight can have significant impact on overall productivity [10]. Birth weight was found to have a moderate and positive genetic correlation with lamb survival and it should be used as an indirect selection criterion to improve survival for Horro sheep [3].

High and positive genetic correlation for birth weight with average daily gain and moderate positive genetic correlation for birth weight with weaning weight indicated that the selection based on birth weight over a long period could result in improve average daily gain and increase weaning weight. The wider range of lamb breeding values for all traits concluded that, selection of lambs for the next generation would lead to higher genetic progress in the herd [18]. Generally, the presence of strong correlations in some cases indicates that we can select for one of the traits and improvement will be expected in both traits.

\subsubsection{Improvement of Reproductive Traits}

Estimate of heritability under different models have shown that litter size has low heritability (0.063 to 0.167$)$ and the correlation between direct and maternal additive genetic effect is negative $(-0.679)$ for Horro sheep. Thus genetic improvement for this trait could be difficult. As a result, improving the weight of ewes at mating could make sizable increase in litter size. In this regard, the result of this study shows that there could be about $2.5 \%$ additional lamb for $1 \mathrm{~kg}$ increase in flock average weight at mating [4].

Genetic correlations among birth weight, weaning weight, twinning and number of lambs at weaning were in the range of 0.57 to 0.86 for Horro sheep [3]. Twinning was found to have medium heritability and repeatability, and moderate to high genetic correlation with number of lambs weaned, birth weight, and weaning weight. These suggest that twinning can be used as a selection criterion for improvement in productivity despite increase in lamb mortality with increase in twinning [3].

\subsection{Development of Breeding Program and Management Schemes}

Commonly cited obstacle to the design and implementation of conservation-based selective breeding programs 
in the tropics is the lack of estimates of genetic parameters to predict genetic gains [22]. Therefore, Information of genetic parameter estimation for different traits is useful in formulating breeding program since these parameters determine the direction and magnitude of genetic improvement [20]. Understanding the concept and knowing this information is important to improve the management system. For example If the heritability value of the animal for specific trait is very high, the animal can be kept or culled based on the first record of observation otherwise if the repeatability is low, more than one observation on the same character is necessary for a decision on the animal and designing appropriate management system [11]. To conclude this part, estimates of genetic parameters are necessary for genetic evaluation of sheep and for choosing the best selection schemes [32].

\section{Conclusions}

Development of effective genetic evaluation and improvement programs requires knowledge of the genetic parameters (genetic variance of each trait and covariance among traits) for economically important production traits. Accurate estimation of these genetic parameters requires data to be corrected to accommodate differences in known environmental effects that influence the growth performance of livestock. The contribution of these environmental effects to the total phenotypic variation should be minimized before estimating the genetic parameters. Thus, adjustment of performance records for known environmental effects has an important role in reducing the non-genetic or environmental components of phenotypic variance. Genetic relationships between the different traits are also required to make appropriate decisions related to selection and to estimate the response to selection. More accurate estimates of genetic parameters and in particular correlations between economically important traits are required for accurate genetic evaluation and development of breeding objectives. Estimates of genetic parameters from different studies suggest that there exists substantial additive genetic variability in the population. The genetic trends confirm that selective breeding can lead to significant genetic improvement in different sheep breeds.

Estimates under different models have shown that reproductive traits have low heritability estimate, thus genetic improvement through direct selection for those trait would be slow because female reproductive traits were highly influenced by the environment. So, it could be more improved through manipulation of production management to reduce environmental influence. Whereas for productive traits having high direct heritability estimate due to the high genetic variances, there is high possibility of improvement through selection. Except for Blackhead Somali sheep breed which has high genetic correlation between birth weight and weaning weight, Horro, Menz and Afar sheep have high genetic correlation between post-weaning weight traits. Therefore, indicating selection for one trait improves the other trait in those sheep breeds. In order to develop alternative improvement program for sheep breeds in Ethiopia, estimates of genetic parameters for economical growth and reproduction traits should be done by design experiments since the information on most sheep breed is nonexistence. From the previous studies, it can be concluded that medium to high direct and maternal heritability estimates obtained suggested that the selection would be an effective means of genetic improvement for early growth traits for Horro and Menz sheep through well-designed selection programmes.

\section{References}

[1] Gowane, G.R., Chopra, A., Prince, L.L.L., Paswan, C. and Arora, A.L. (2010) Estimates of (Co)Variance Components and Genetic Parameters for Body Weights and First Greasy Fleece Weight in Bharat Merino Sheep. Animal, 4, 425-431. http://dx.doi.org/10.1017/S1751731109991157

[2] Nagy, I., Sölkner, J., Komlósi, I. and Sáfár, L. (1999) Genetic Parameters of Production and Fertility Traits in Hungarian Merino Sheep. Journal of Animal Breeding and Genetics, 116, 399-413. http://dx.doi.org/10.1046/j.1439-0388.1999.00204.x

[3] Abegaz, S. (2002) Genetic Evaluation of Production, Reproduction and Survival in a Flock of Ethiopian Horro Sheep. Ph.D. Dissertation, Faculty of Natural and Agricultural Sciences Department of Animal, Wildlife and Grassland Sciences University of the Free State, Bloemfontein, 118 p.

[4] Abegaz, S. and Duguma, G. (2000) Genetic and Phenotypic Parameters of Growth, Reproductive and Survival Performance of Horro Sheep at Bako Agricultural Research Center. Research Fellowship Report, International Livestock Research Institute (ILRI), Addis Ababa.

[5] F.G. Kesbi, Eskandarinasab, M. and Hassanabadi, A. (2008) Estimation of Genetic Parameters for Lamb Weight at Various Ages in Mehraban Sheep (Short Communication). Italian Journal of Animal Science, 7, 95-103.

[6] Matika, O., Van Wyk, J.B., Erasmus, G.J. and Baker, R.L. (2003) Genetic Parameter Estimates in Sabi Sheep. Live- 
stock Production Science, 79, 17-28.

[7] Abegaz, S., Negussie, E., Duguma, G. and Rege, J.E.O. (2002) Genetic Parameter Estimates for Growth Traits in Horro Sheep. Journal of Animal Breeding and Genetics, 119, 35-45. http://dx.doi.org/10.1046/j.1439-0388.2002.00309.x

[8] Juma, K.H. and Alkass, J.E. (2006) Genetic and Phenotypic Parameters of Some Economic Characteristics in Awassi Sheep of IRAQ: A Review. Egyptian Journal of Sheep, Goat and Desert Animals Sciences, 1, 15-29.

[9] Sang Hyon, O.H. (2003) Estimation of Genetic Parameters for Boar Semen Traits. PhD. Dissertation, Faculty of North Carolina State University, Raleigh.

[10] Yacob, Y. (2008) Environmental and Genetic Parameters of Growth, Reproductive and Survival Performance of Afar and Blackhead Somali Sheep at Werer Agricultural Research Center. Fellowship Report, International Livestock Research Institute (ILRI) and Ethiopian Institute of Agricultural Research (EIAR), Ethiopia.

[11] Kanakaraj, P. (2001) A Text Book on Animal Genetics. Army Printing Press, Lucknow.

[12] Clément, V., Bibé, B., Verrier, É., Elsen, J.M., Manfredi, E., Bouix, J. and Hanocq, É. (2001) Simulation Analysis to Test the Influence of Model Adequacy and Data Structure on the Estimation of Genetic Parameters for Traits with Direct and Maternal Effects. Genetics Selection Evolution, 33, 369-395. http://dx.doi.org/10.1186/1297-9686-33-4-369

[13] Matos, C.A., Thomas, D.L., Gianola, D., Tempelman, R.J. and Young, L.D. (1997) Genetic Analysis of Discrete Reproductive Traits in Sheep Using Linear and Nonlinear Models: I. Estimation of Genetic Parameters. Journal of Animal Science, 75, 76-87.

[14] Ozder, M., Sezenler, T., Refik, A. and Ceyhan, A. (2009) Genetic and Non-Genetic Parameter Estimates for Growth Traits in Turkish Merino Lambs. Journal of Animal and Veterinary Advances, 8, 1729-1734.

[15] Rao, S. (1997) Genetic Analysis of Sheep Discrete Reproductive Traits Using Simulation and Field Data. PhD. Dissertation, Faculty of the Virginia Polytechnic Institute and State University, Department of Animal and Poultry Science, Blacksburg.

[16] Graser, H.U., Smith, S.P. and Tier, B. (1987) A Derivative-Free Approach for Estimating Variance Components in Animal Models by Restricted Maximum Likelihood. Journal of Animal Science, 64, 1362-1370.

[17] Reverter, A., Kaiser, C.J. and Mallinckrodt, C.H. (1998) A Bootstrap Approach to Confidence Regions for Genetic Parameters from Method R Estimates. Journal of Animal Science, 76, 2263-2271.

[18] El-Arian, M.N., Salama, M.A. and Mohamed, H.A. (2008) Estimation of Genetic Parameters and Breeding Value for Growth Traits on Romanov Lambs in Egypt. Mansoura University Journal of Agricultural Sciences, 33, 2569-2576.

[19] Chrilukovian, W. (2006) Genetic Evaluation of Growth and Reproductive Performance of the Kenya Boran Cattle. M.Sc. Thesis, Production (Animal Breeding Option) of Egerton University, Njoro.

[20] Haile, A. (2006) Genetic and Economic Analysis of Ethiopian Boran Cattle and Their Crosses with Holstien Friesian in Centeral Ethiopia. Ph.D. Dissertation, National Dairy Research Institute, Deemed University, Karnal.

[21] Hermiz, H.N., Alkass, J.E., Hobi, A.A. and Asofi, M.K. (2009) Genetic and Phenotypic Parameters of Body Weights in Iraqi Local Goat and Their Crosses with Damascus. Journal of Dohuk University, 12, 189-194.

[22] Gizaw, S., Lemma, S., Komen, H. and van Arendonk, J.A.M. (2007) Estimates of Genetic Parameters and Genetic Trends for Live Weight and Fleece Traits in Menz Sheep. Small Ruminant Research, 70, 145-153. http://dx.doi.org/10.1016/j.smallrumres.2006.02.007

[23] Hassen, Y., Fuerst-Waltl, B. and Sölkner, J. (2003) Genetic Parameter Estimates for Birth Weight, Weaning Weight and Average Daily Gain in Pure and Crossbred Sheep in Ethiopia. Journal of Animal Breeding and Genetics, 120, 2938. http://dx.doi.org/10.1186/1297-9686-24-6-487

[24] Bradford, G.E., Sakul, H., Neira, R., Dally, F.M.R. and Finley, C.M. (1994) Reproduction in Sheep Selected for Weaning Weight or Litter Size in a Range Environment. Proceedings of 5th World Congress on Genetics Applied to Livestock Production, 18, 95

[25] Gizaw, S. and Joshi, B.K. (2004) Genetic and Non-Genetic Factors Affecting Survivability of Menz and Awassi $\times$ Menz Crossbred Sheep in Ethiopia. Indian Journal of Animal Sciences, 74, 887-889.

[26] Fogarty, N.M. (1995) Genetic Parameters for Live Weight, Fat and Muscle Measurements, Wool Production and Reproduction in Sheep: A Review. Animal Breeding Abstracts, 63, 101.

[27] Al-Shorepy, S.A. (1995) Selection to Improvement Spring Fertility in a Crossbred Sheep Population. Ph.D. Dissertation, Virginia Polytechnic Institute \& State University, Blacksburg.

[28] Meyer, K. (1992) Bias and Sampling Covariances of Estimates of Variance Components Due to Maternal Effects. Genetics Selection Evolution, 24, 487-509. http://dx.doi.org/10.1186/1297-9686-24-6-487

[29] Vatankhah, M., Talebi, M.A. and Edriss, M.A. (2008) Estimation of Genetic Parameters for Reproductive Traits in 
Lori-Bakhtiari Sheep (Short Communication). Small Ruminant Research, 74, 216-220. http://www.sciencedirect.com/ http://dx.doi.org/10.1016/j.smallrumres.2007.02.008

[30] Falconer, D.S. (1989) Introduction to Quantitative Genetics. 3rd Edition, Longman, London.

[31] Atkins, K.D. (1986) A Genetic Analysis of the Components of Lifetime Productivity in Scottish Blackface Sheep. Animal Production, 43, 405-419. http://dx.doi.org/10.1016/S0921-4488(01)00256-5

[32] Rosati, A., Mousa, E., Van Vleck, L.D. and Young, L.D. (2002) Genetic Parameters of Reproductive Traits in Sheep. Small Ruminant Research, 43, 65-74. http://dx.doi.org/10.1016/S0921-4488(01)00256-5 